\title{
GIUSEPPE CASTIGLIONE (LANG SHINING), PRECURSOR DE LA PRIMERA MUNDIALIZACIÓN PICTÓRICO-ARQUITECTÓNICA
}

\author{
MANUEL V. CASTILLA \\ Universidad de Sevilla
}

El arte tiene dos principios, la idea que produjo el arte europeo y la quimera que produjo el arte oriental.

VICTOR HugO

\section{Introducción}

La decadencia del Renacimiento, a finales del siglo XVI, significó una ruptura desde un punto de vista pictórico, escultórico y arquitectónico. A partir de ese momento, los nuevos esquemas compositivos eliminaron algunos elementos tradicionales en las construcciones de tipo religioso, y permitieron la realización de composiciones espaciales, sumamente complejas en algunos casos, aunque su dinamismo resultaba sorprendente. Se produjo una explosión generalizada de todas las artes: pintura, cerámica, escultura y arquitectura, donde la perspectiva matemática lineal jugó un papel prominente.

Los orígenes de la perspectiva se remontan a los murales romanos ilusionistas, integrados dentro de la propia arquitectura, pero su verdadero impulso surgió en siglo XIV con Filippo Brunelleschi (1377-1446), que la desarrolló formalmente como perspectiva matemática lineal y la relacionó y utilizó en su obra arquitectónica. Leonardo da Vinci (1452-1519) destacó su im-

Este artículo fue recibido por la dirección de la revista el 26 de mayo de 2015 y aceptado para su publicación el 6 de julio de 2015. 
portancia en la consolidación de la interpretación matemática de la realidad: "La Pintura se cimienta en la Perspectiva, que no es más que el arte de representar lo que se ve, o sea, de hacer objetos parecidos a los que contemplamos con los ojos". ${ }^{1}$ En palabras de M. Kemp, la perspectiva es el arte de "crear una ilusión sistemática de formas que se degradan más allá de la superficie plana de una mesa, de una tela, de una pared o de un techo". ${ }^{2}$

Entre los grandes maestros que utilizaron esta forma de expresión artística destaca Anibal Carracci (1560-1609), autor de una de las obras maestras de pintura italiana del siglo XVII; me refiero a la bóveda de la galería del Palacio Farnesio (1597-1604), sobre el triunfo del amor (Amor Omnia Vincit).

A. Carracci, en una falsa arquitectura de gran complejidad, generó un conjunto de escenas mitológicas con atrevido dinamismo. Esta obra influyó en varias generaciones de decoradores clásicos y barrocos.

Los contrastes luminosos y, a veces, la difusa luminosidad, las composiciones dinámicas, los efectos de ilusionismo y la abundante fastuosidad, marcan algunas características de la obra de otro gran protagonista, Giovanni Lanfranco (1582-1647), mientras que los trabajos y las publicaciones del jesuita Andrea Pozzo (1642-1709) ${ }^{3}$ tuvieron una extraordinaria influencia en la perspectiva arquitectónica y en la arquitectura propiamente dicha. Conocedor de la perspectiva matemática renacentista, interpretó el espacio tridimensional como lo perciben nuestros sentidos. Es justo también destacar el trabajo del genovés Giovanni Battista Gaulli (1639-1709), que transformó "Il Gesú” en iglesia barroca, con su representación de la glorificación de Jesús en la bóveda, a la que dio una solemne grandiosidad. Podríamos decir que Pozzo y Gaulli, con sus técnicas ilusionistas, funden el espacio pictórico con el espacio real y convierten lo fantástico en realidad. Con estos antecedentes,

${ }^{1}$ Esta cita de Leonardo está recogida en J. L. Ferrer Muñoz, La perspectiva en las artes y en la técnica, Valencia, Universidad Politécnica de Valencia, 1981, p. 81.

${ }^{2}$ Martin Kemp, La scienza dell'arte. Prospettiva e perzecezione visiva da Brunelleschi a Seurat, trad. F. Camerota, Florencia, Giunti, 1994, p. 15.

${ }^{3}$ Pintor italiano y maestro en el arte y la ciencia de la perspectiva. Para una completa revisión de su biografía, véase Vittorio de Feo y Vittorio Martinelli, Andrea Pozzo, Milán, Electa, 1996. 
parece lógica la relación de la pintura barroca con la fantasía, la imaginación y el exotismo arquitectónico.

En la arquitectura existieron dos corrientes: Bernini (15981680) y Borromini (1599-1667). En la primera de ellas, la arquitectura tuvo un papel secundario, frente a la pintura y la escultura de tipo ilusionista. En Borromini, la creación arquitectónica es un modo de expresión emocional. Su sorprendente imaginación y creatividad fue señalada por algunos contemporáneos como corruptora de la arquitectura, pero su obra marcaría los primeros intentos sobre una composición espacial dinámica, que buscaba sorprendentes espacios escénicos. La obra de su discípulo Guarino Guarini (1538-1612), ${ }^{4}$ vista por algunos investigadores como continuación de la extraña rareza de Borromini, permitió el nacimiento de un estilo arquitectónico encuadrado en el fantasticare. ${ }^{5} \mathrm{Su}$ originalidad, imaginación y fantasía, lo llevaron al desarrollo de geometrías complejas claramente innovadoras.

Toda esta fuerza de renovación cultural y artística fue aprovechada por los misioneros, fundamentalmente jesuitas, que en su papel de profesores y asesores científicos, pintores y arquitectos de la corte imperial, introdujeron la perspectiva matemática lineal en China.

El emperador Kangxi (1654-1722), amante de la ciencia europea, tuvo un gran protagonismo en la introducción del arte de la perspectiva, y la presencia del jesuita G. Gherardini (16551723), que había tenido como referentes a Michelangelo Colonna (1604-1687) y Agostino Mitelli (1609-1660), dio un impulso definitivo al conocimiento de esta técnica pictórica. Colonna y Mitelli fueron dos experimentados maestros en grandes formatos de pintura ilusionista quienes simulaban arquitecturas mediante la técnica de la quadratura. ${ }^{6}$ En este contexto, la obra de Andrea Pozzo, Perspectiva Pictorum et Architectorum, que había sido traducida a diferentes idiomas y adaptada a los fundamentos

${ }^{4}$ La obra de Guarino Guarini está perfectamente analizada en Harold Alan Meek, Guarino Guarini and his Architecture, New Haven-Londres, Yale University, 1989.

${ }^{5}$ Este estilo profundiza en el estudio de secuencias espaciales; toma especial relevancia la definición geométrica. Básicamente, el punto de partida es un octógono en planta, que en ocasiones evoluciona hasta el óvalo.

${ }^{6}$ Ingrid Sjöström, Quadratura: Studies in Italian Ceiling Painting, Estocolmo, Universidad de Estocolmo, 1978. 
y las formas de la tradición china, representó el punto de partida de la difusión de la perspectiva en Asia Oriental.

La salida de G. Gherardini, de Beijing, se produjo en 1704, pero el emperador Kangxi pudo mantener activos los trabajos de quadratura con la llegada, en 1715, del hermano lego jesuita Giuseppe Castiglione. Hasta entonces, ningún artista chino había empleado las técnicas del chiaroscuro ni manifestado influencias renacentistas respecto de la perspectiva. Un discípulo de G. Castiglione, Nian Xiyao, publicó, en 1735, el primer tratado chino sobre perspectiva y pintura ilusionista de estilo europeo, titulado Ciencia de la visión (Shixue). ${ }^{7}$ Esta obra fue muy utilizada por los eruditos de la corte para intentar un acercamiento a las técnicas europeas de la perspectiva. Es evidente que Castiglione tuvo una influencia decisiva en la publicación del Shixue y en la utilización y difusión de la perspectiva matemática lineal, al introducir en China algo que los chinos desconocían: la "sombra".

En este sentido, Elisabetta Corsi escribe:

Todavía activo [Nian Xiyao] a los sesenta y dos años de edad, estaba por publicar la segunda edición de su Shixue, la "Ciencia de la visión", una traducción parcial del famoso tratado de Andrea Pozzo, S. J. [...], Perspectiva Pictorum et Architectorum (1693, 1770). Ya había publicado una edición en 1729, titulada Shixue jingyun, "Esencia de la ciencia de la visión", pero ahora, al considerar el asunto otra vez, sintió que su deber era sacar a la luz una segunda versión aumentada. ${ }^{8}$

La traducción de la obra de A. Pozzo fue realizada con la ayuda de Giuseppe Castiglione, que, como veremos, dejó una huella imborrable en los territorios asiáticos como maestro, pintor, arquitecto y misionero. De su obra interesan dos aspectos que están relacionados con algunos de los fundamentos aplicables al término actual de mundialización: su original bibridación y su tendencia globalizadora.

${ }^{7}$ Flora Botton Beja, "Reseña del libro de Elisabetta Corsi, La fábrica de las ilusiones. Los jesuitas y la difusión de la perspectiva lineal en China (1698-1766)", Estudios de Asia y África, vol. XL (2), núm. 127, 2005, pp. 465-469.

${ }^{8}$ Elisabetta Corsi, "Perspectiva iluminadora e iluminación de la perspectiva. La versión del arte occidental de la perspectiva de Nian Xiyao (1671-1738) en los prólogos a la “Ciencia de la visión'”, Estudios de Asia y África, vol. xxxvi (3), núm. 116, 2001, p. 376. 


\section{Hibridación artística}

Giuseppe Castiglione nació en Milán el 19 de julio de 1688. Tras haber realizado el noviciado en el monasterio jesuita de Coímbra (Portugal), navegó en un galeón portugués hasta llegar a Macao, en julio de 1715. Cuatro meses más tarde fue presentado al emperador, bajo la protección del padre Matteo Ripa (16821746). Su conocimiento de la lengua china le permitió servir como intérprete de la corte y ejercer de pintor y arquitecto; trabajó junto a los artistas asiáticos y recibió el nombre chino de Lang Shining. ${ }^{9}$ Su llegada a la corte Qing le dio la oportunidad de comenzar una nueva forma de evangelizar, a través de las ciencias y las artes, y cumplir así con sus obligaciones misioneras. El jesuita trabajó en la corte imperial desde 1715 hasta su muerte, en 1766, para los emperadores Kangxi (1654-1722), Yongzheng (1678-1739) y Qianlong (1711-1799), y la poderosa razón de su presencia en China la explica Marco Musillo: "Kangxi había solicitado un pintor europeo y los jesuitas no quisieron defraudarlo". ${ }^{10}$ Castiglione, que había sido discípulo del quadraturista Andrea Pozzo, ${ }^{11}$ fue el primer artista que articuló la pintura y la arquitectura de estilo europeo con objetivos muy concretos. El jesuita inyectó nuevas ideas en los artistas de la corte Qing, y las combinó con las propiamente chinas, con lo que abrió nuevas líneas de actuación pictórica y arquitectónica entre Asia Oriental y Occidente.

En este contexto es posible que pueda parecer poco fundamentado relacionar la obra de un artista jesuita que vivió

${ }^{9}$ Sobre la educación artística, familiar y misionera de G. Castiglione existen distintos ensayos y referencias bibliográficas. Véanse de Marco Musillo: "Bridging: Europe and China: The Professional Life of Giuseppe Castiglione (1688- 1766)", tesis de doctorado, University of East Anglia, Norwich, 2006, y "Reconciling Two Careers: The Jesuit Memoir of Giuseppe Castiglione Lay Brother and Qing Imperial Painter”, Eighteenth-Century Studies, vol. 42, núm. 1, 2008, pp. 45-59. Y de Michèle Pirazzoli-t'Serstevens, Giuseppe Castiglione 1688-1766: Peintre et architecte à la cour de Chine, París, Thalia, 2007.

${ }^{10}$ Musillo, Bridging: Europe and China, op. cit., p. 24.

${ }^{11}$ A. Pozzo nació en Trento, en 1642, y se formó en Como y Milán, donde ingresó en la Compañía de Jesús. Autor de Prospettiva de’ pittori e architetti (moderna edición Italo Svevo, Roma, 2003), difundió la influencia del barroco por toda Europa. Se le recuerda como un artista extraordinariamente versátil, ya que trabajó como arquitecto, pintor, decorador y teórico del arte. 
hace más de tres siglos, con un acontecimiento relativamente moderno como es el fenómeno de la mundialización. Aquellas reflexiones que ayuden a razonar sobre la existencia de elementos globalizadores en su obra pictórica y arquitectónica serán posibles pruebas de su papel precursor en la mundialización del arte. Desde este punto de vista, y teniendo en cuenta que en la obra del jesuita existen avances artísticos de lo que ocurrió más tarde, Castiglione puede ayudarnos a interpretar algunas vertientes artísticas de los siglos XIX y Xx. Por otro lado, la conexión entre su concepción de la perspectiva pictórico-arquitectónica y el concepto de mundialización puede no ser suficiente para explicar el objetivo de este trabajo, pero sí explicaría el paralelismo entre sus ideas y algunas líneas artísticas modernas. El artista jesuita colmó las aspiraciones y los deseos del emperador desde el punto de vista artístico, y esto le permitió adquirir una formación capaz de transmitir esos mismos deseos y sentimientos a futuras generaciones en distintas situaciones geográficas; pero no es fácil probar la influencia del mundo de las ideas y los conocimientos en las realidades artísticas que ocurren un tiempo después. Esto permitiría admitir que los conceptos creativos de Castiglione coinciden con los que inspiran la modernidad. Sin embargo, existe un hecho cierto: el artista jesuita estaba convencido de que su pasión evangelizadora era el camino perfecto para la hibridación artística entre China y Europa. Sin conocer su papel de precursor de una posible mundialización artística, Castiglione siempre tuvo claro que el acercamiento a China era ante todo una misión evangélica, basada en el intercambio de las ciencias y las artes. La promoción de ambas inquietudes fue para él una continua meta para extender y acercar a Asia y Europa. Su trinomio inculturador, ciencia-arte-religión, siempre estuvo fundamentado en una base científica, un propósito artístico y una finalidad evangelizadora. Esta convergencia de intereses no es ajena al principal objetivo del jesuita misionero: conectar su obra con la presencia de Dios como fuente de realidad geométrica a través de sus pinturas y proyectos arquitectónicos. La evangelización como consecuencia del Concilio de Trento $(1545-1563)$ y su pasión por las artes fueron sus puntos de partida, defendidos incluso por encima de los decretos imperiales contra la religión católica. En alguna ocasión, ante una situa- 
ción incómoda, Castiglione respondió al emperador Qianlong: "No, señor, pero me encuentro en gran estado de desánimo [...] Su Majestad ha condenado nuestra sagrada religión. Las calles están llenas de carteles que la proscriben. ¿Cómo seremos de esta manera capaces de servir a su Majestad en paz? Cuando los europeos tengan conocimiento de esta orden dada, ¿habrá alguien que venga a servirle?". ${ }^{12}$

La secuencia de fenómenos y manifestaciones ocurridos desde su presencia en la actividad científico-artístico-religiosa supone una prueba de lo que se intenta sugerir en este trabajo. Así, muchos de los aspectos que intervienen en la mundialización se dieron en las decisiones artísticas del jesuita, que tienen un parecido con las que encontramos en la actualidad en China y Europa. Si la mundialización puede concebirse como una "tendencia hacia la globalización", el motor de esa tendencia tuvo que ser activado por las fuentes que la idearon y la propusieron. Esto no quiere decir que algunos aspectos globalizados de la pintura y la arquitectura sean realmente así debido al ingenio del misionero. Pero, paralelamente, hay que constatar la coincidencia de su portentosa transformación intelectual con la hibridación cultural chino-europea que conocemos en la actualidad. Este ejemplo de síntesis entre China y Europa nos demuestra que la mundialización artística va unida al devenir de las civilizaciones a partir de una causa motora. Quizá no podamos razonar suficientemente que la concepción pictórico-arquitectónica de Castiglione tenga un claro potencial globalizador, pero sí cabe identificar su parecido con algunas tendencias artísticas recientes; con su trabajo en la corte de Qianlong sembró la semilla de la ciencia y el arte barroco en el fértil país asiático.

${ }^{12}$ Joselyn M. N. Marinescu, "Defending Christianity in China: The Jesuit Defense of Christianity in the Lettres édifiantes et curieuses \& Ruijianlu in relation to the Yongzheng Proscription of 1724", tesis de doctorado, Kansas State University, Manhattan, 2008, p. 250. 


\section{Pintura}

Castiglione no sólo utilizó sus conocimientos europeos, sino que adoptó conceptos, temas y materiales chinos en la ejecución de sus obras. Y todo ello a pesar de que los chinos, que poseían una larga tradición "cosmológica”, "realista” y "miniaturista”, ignoraban que sus obras estaban a una gran distancia del estilo naturalista europeo. La pintura china consistía simplemente en la representación de escenas rituales, históricas o bucólicas, cargadas de simbolismo. Aceptada esta importante influencia en la metodología artística del jesuita, su éxito se basó además en utilizar conjuntamente conceptos de óptica, geometría, matemáticas y pintura. En esta estrategia fundamentó su extraordinaria originalidad. Sus formas y conceptos básicos de la perspectiva matemática lineal, alimentados con la aportación de elementos básicos de la pintura china, los transmitió a otros jesuitas y alumnos de la corte, donde ejercían un buen número de pintores y matemáticos chinos. Generó así una forma de hibridación que se extendió a todas sus representaciones artísticas.

La técnica que los artistas jesuitas utilizaron en la transformación china de la perspectiva matemática lineal europea fue el llamado método de líneas o xianfa, ${ }^{13}$ directamente relacionado con la profundidad y el escorzo, creado a través de líneas que convergen en un punto focal (punto de fuga). En este sentido, xianfa puede definirse como el encuentro de la perspectiva matemática lineal europea con la influencia del espíritu del arte chino. Inicialmente, Castiglione se inclinaba de forma natural hacia la perspectiva occidental, pero la lenta y gradual transformación de su expresión artística fundamentó una vigorosa hibridación chino-europea, como comprobaremos al comparar alguna de sus obras con otras de artistas chinos. Paulatinamente, temas chinos como la caza (figura 1), los juegos, las excursiones o los pájaros y, en general, todos los sucesos importantes de la corte, fueron inspirando algunas de sus grandes obras. Michel Beurdeley escribe: "Los europeos, en general, admiraban la preciosidad, el colorido de los pintores de flores

${ }^{13}$ Véase Elisabetta Corsi, “Términos chinos utilizados como sinónimo de 'perspectiva' y ecos del naturalismo en algunos textos sobre pintura del siglo XviII”, Estudios de Asia y África, vol. XxxII (3), núm. 104, 1997, p. 441. 
FiguRA 1. Jinete cazando una liebre, siglos III-IV a.n.e., pintura sobre ladrillo, $17 \times 36 \mathrm{~cm}$, tumba próxima a Chiayukuan, provincia de Kansu

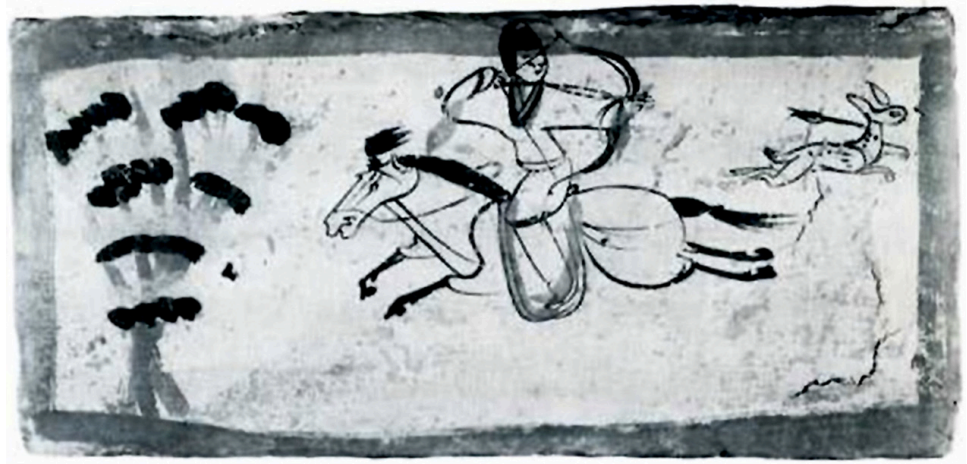

FIGURA 2. Giuseppe Castiglione (Lang Shining), Machang kanzhen (fragmento), 1759, tinta y color sobre papel, National Palace Museum, Taipéi

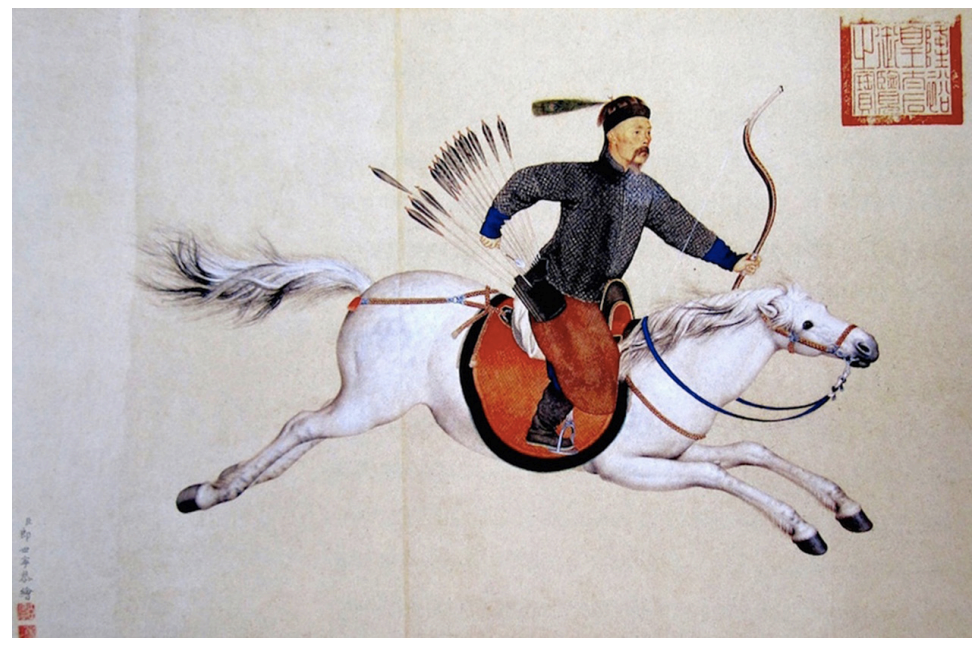

Esta figura puede verse en Pirazoli-t’Serstevens, Giuseppe Castiglione, 1688-1766: Peintre et architecte à la cour de Chine, París, Thalia, 2007, p. 186. 
Figura 3. Zhang Wo, Emperador Tai Yi, detalle de rollo de mano, siglo XII, tinta sobre papel, $28 \times 41 \mathrm{~cm}$, The Shanghai Museum of Art

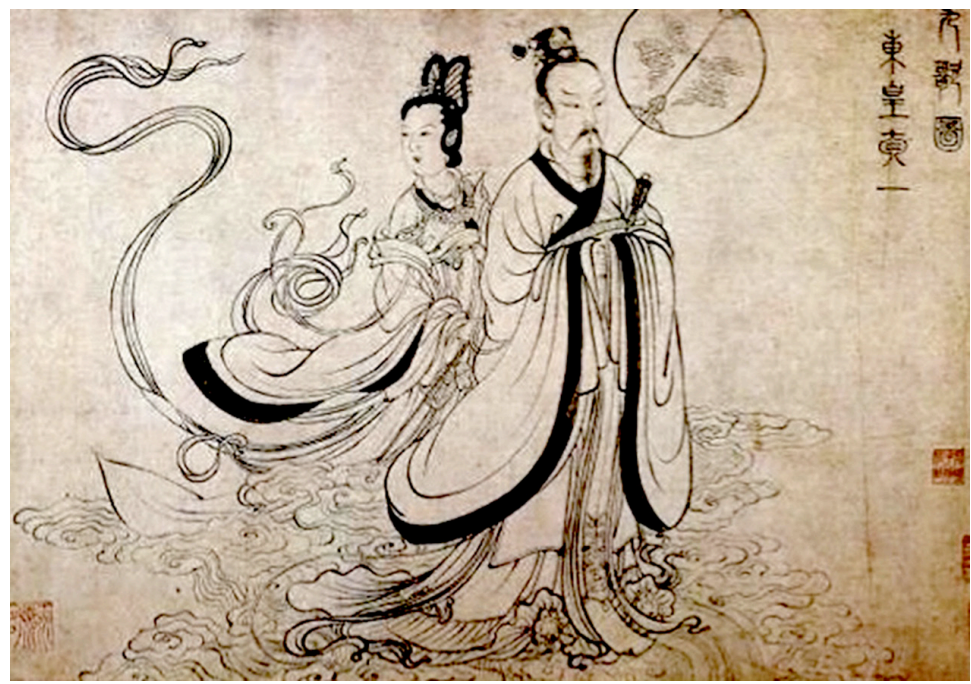

y pájaros chinos, pero se les escapaba el sentimiento poético que se manifiesta siempre en las más cuidadosas de estas representaciones". ${ }^{14}$

La obra pictórica de Castiglione refleja tanto el conocimiento que éste tenía de las prioridades de la pintura china como su acomodo intelectual a ellas, hasta adquirir un alto grado de hibridación artística. En este sentido, el artista demuestra un extraordinario conocimiento de la técnica kung- pi (trabajopincel), en la que están presentes los delicados contornos y la precisión de la imagen, ejecutada en soportes de escasa absorción (seda o papel tratado). En el retrato, género que se cultivó profusamente en la corte de Qianlong, utilizó con frecuencia el trazo homogéneo con tinta concentrada, característico de la pintura china (figura 3), para delinear perfiles y contornos.

${ }^{14}$ Michel Beurdeley y Marco Musillo, Peintres Jésuites en China au XVIII siècle, París, Anthèse, 1997, p. 163. 
Figura 4. Atribuido a Giuseppe Castiglione (Lang Shining), Retrato de Qianlong, 1735 o 1736, rollo vertical, tinta y color en seda, The Palace Museum, Beijing

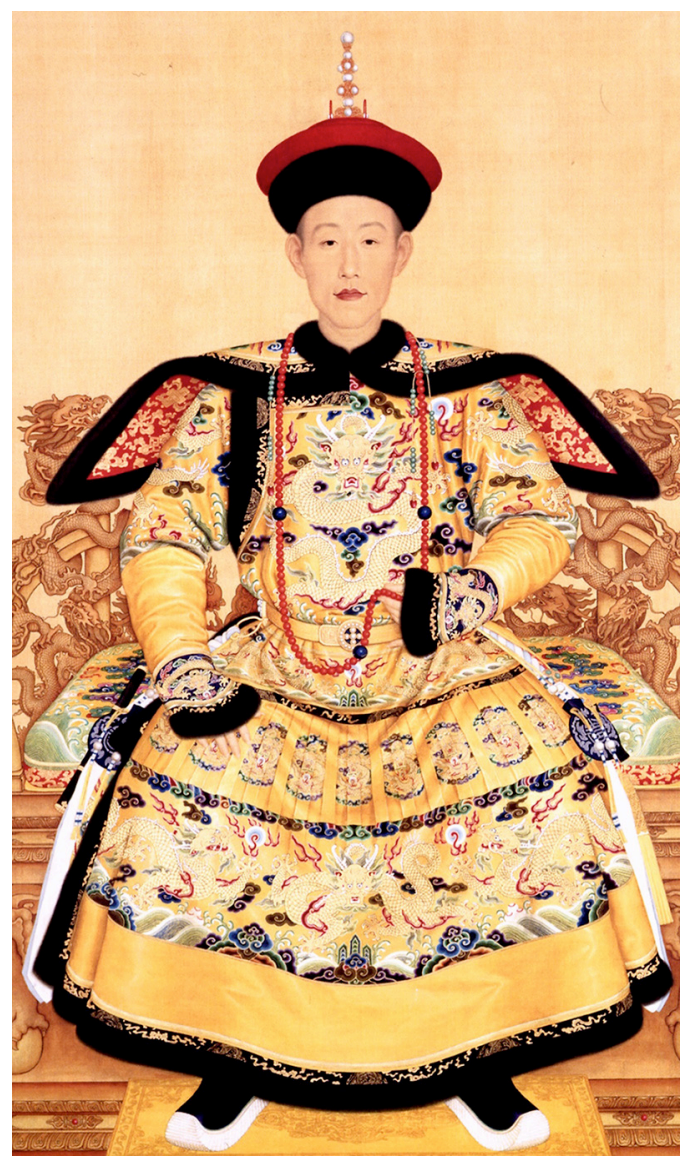

Las múltiples posibilidades expresivas del trazo, desde su vertiente más contenida hasta su manifestación más atrevida, fueron magistralmente manejadas por Castiglione en su pintura (figura 4). Otro de los temas que Castiglione desarrolló en su pintura cortesana fueron los caballos, impulsado quizá porque Qianlong los adoraba. Incluso llegó a dominar métodos 
FIGURA 5. Giuseppe Castiglione (Lang Shining), Huadixianmang to (fragmento del rollo), Ping'an chun xin tu (Mensajes de una primavera pacífica), hacia 1735 , rollo vertical, tinta y color sobre seda, The Palace Museum, Beijing

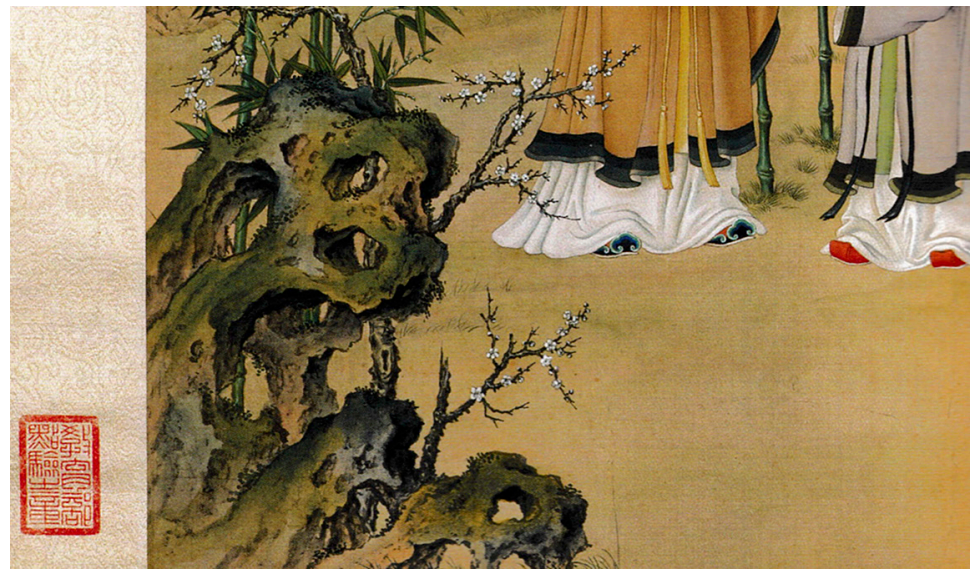

pictóricos chinos, como el de los llenos y los vacíos, o el de los triángulos rectángulos. ${ }^{15}$ Castiglione utilizó el vacío para dar la impresión de que había un fondo detrás de la figura, y anticipar la presencia de un espacio real y profundo. De esta forma, la imagen que quiere representar se genera y se reconoce como tal, y la percepción del espectador es la de una pintura superpuesta a un fondo, y un espacio que la envuelve. A medida que avanza la vista del espectador desde los primeros planos hasta los más lejanos, los espacios llenos y vacíos se intercalan para generar un dinámico y magistral equilibrio.

Esta forma expresiva de la pintura china constituye una de las aportaciones más significativas al arte de Castiglione (figura 5). Incluso, si se observan las hojas de bambú existentes en esta pintura, se comprueba que están resueltas con trazos únicos de pincelada directa, con claras influencias de la pintura

${ }^{15}$ Véase Corsi, “Términos chinos utilizados como sinónimos...”, op. cit., p. 441. 
T'chan. ${ }^{16}$ Es anecdótico destacar que en muchas de sus obras aparece un elemento conocido como pequeño sello personal, cuya utilización por los artistas chinos para firmar e identificar pinturas se remonta al reinado del emperador T'ai-Tsung de la dinastía Tang (618-906)..$^{17}$

Parece que aún queda un largo camino por recorrer para interpretar el arte de Giuseppe Castiglione como motor de una mundialización artística, pero sus diseños pictórico-arquitectónicos han sido referentes compositivos en territorios muy diversos.

\section{Arquitectura}

Es justo destacar que el emperador Qianlong influyó mucho en la obra del jesuita, como conocedor de lo más científico y artístico de la corte, y más concretamente como patrocinador personal del pintor italiano. Durante el reinado del emperador Qianlong, los artistas de la corte china y los europeos colaboraron para crear nuevos caminos en el campo de las ciencias y las artes; combinaron técnicas importadas de Europa con técnicas chinas, y crearon pinturas ilusionistas y diseños arquitectónicos de inusitada belleza, para la recreación de Qianlong. La extraordinaria y larga relación entre el emperador Qianlong y G. Castiglione llevó nuevas formas pictóricas y arquitectónicas europeizantes en la China Qing.

Precisamente, la vertiente arquitectónica del jesuita generó una inquietud artística capaz de preludiar algunas corrientes desarrolladas muchos años después, que pueden haber influido en otras desarrolladas en la actualidad. Nos referimos a su labor como arquitecto-diseñador de un mágico proyecto arquitectónico en los jardines del Yuanming Yuan: los palacios de estilo europeo (Xiyang Lou). La construcción de estos recintos palaciegos de estilo occidental (figura 6) comenzó en 1747 por

${ }^{16} \mathrm{La}$ pintura T'chan construye la imagen con trazos rápidos y directos, pues apelan a la soltura del artista y a una fuerte aplicación caligráfica del pincel. Véase Blas Sierra de la Calle, Catálogo Colección S. C. Cheng de Arte chino, Valladolid, Museo Oriental-Caja España, 1999, p. 92.

${ }^{17}$ Ibid., pp. 20-27. 
Figura 6. Plano de los palacios de estilo europeo

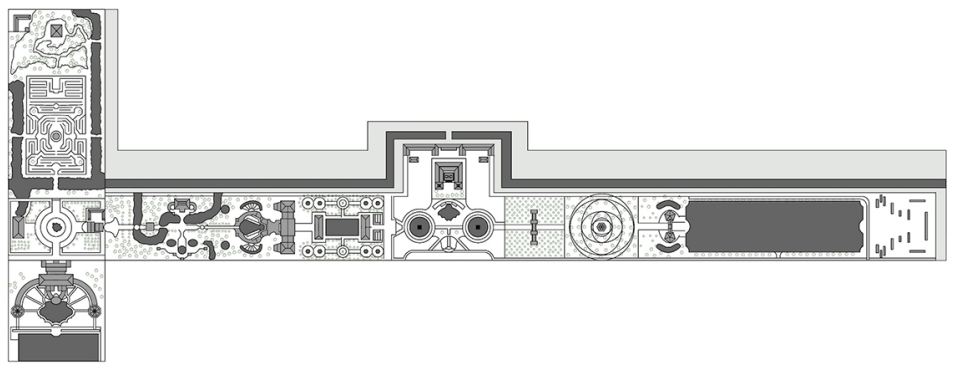

orden del emperador Qianlong en terrenos situados al norte de los Jardines de la Perfecta Brillantez (Yuanming Yuan) en el noroeste de Beijing. Los diseños de G. Castiglione fueron realizados en un barroco fascinante, ${ }^{18}$ algunos elementos ornamentales y arquitectónicos, en cierta forma, recordaban la arquitectura de Guarino Guarini y Borromini. ${ }^{19}$ Su estilo constructivo combinaba formas arquitectónicas chinas y europeas. Las estructuras se hicieron de madera china, incluidas las columnas; los recubrimientos eran de piedra gris y mármol blanco; las columnas exteriores, escaleras y esculturas se hicieron al modo europeo. Existían además elementos constructivos en los que estaban presentes las tradiciones chinas y europeas a la vez, como sucedía en las cerámicas vidriadas de diferentes colores. En la decoración exterior de algunos palacios se aplicó el estilo rococó, floreciente en el sur de Europa.

En este sentido, Young-Tsu Wong, ${ }^{20}$ conocido experto en el Yuanming Yuan, manifiesta que no sólo contiene palacios barrocos de estilo italiano, sino que además la distribución del espacio era de corte occidental. Por su parte, Jin Yufeng, ${ }^{21}$ en su

${ }^{18}$ Véase Cécile Beurdeley y Michel Beurdeley, Giuseppe Castiglione. A Jesuit Painter at the Court of the Chinese Emperors, trad. Michael Bullock, Rutland-Tokio, Tuttle y Co., 1971, pp. 45, 59 y 66-67.

${ }^{19}$ Paul Pelliot, Les Influences Européennes Sur L'art Chinois, París, Imprimerie Nationale, 1928.

${ }^{20}$ Young-Tsu Wong, A Paradise Lost. The Imperial Garden Yuanming Yuan, Honolulú, Universidad de Hawái, 2001, pp. 59-65.

${ }^{21}$ Jin Yufeng, "Yuanming Yuan Xiyang Lou Pingxi (A Critical Analysis of 
artículo "Yuanming Yuan Xiyang Lou Pingxi (A Critical Analysis of the European Section in the Yuanming Yuan), mantiene que los palacios de estilo europeo respetaban los principios del Renacimiento italiano y contenían adornos barrocos y elementos chinos, como los azulejos $l i u l i^{22}$ y las rocas naturales provenientes del lago Taihu. ${ }^{23}$

Es evidente que Castiglione y sus compañeros jesuitas, luego de trascender las barreras culturales, lograron una extraordinaria colaboración entre Asia y Europa, fantásticamente simbolizada en los palacios de estilo europeo del Yuanming Yuan, definidos por Victor Hugo con las siguientes palabras:

Había en un rincón de la tierra, una maravilla del mundo: esta maravilla se llamaba el Palacio de Verano. El arte tiene dos principios, la idea que produjo el arte europeo y la quimera que produjo el arte oriental. El Palacio de Verano era al arte quimérico, lo que el Partenón es al arte ideal [...] Todos los tesoros de todas nuestras catedrales reunidas no igualarían este formidable y espléndido museo de Oriente. ${ }^{24}$

\section{Tendencia globalizadora de la obra pictórico- arquitectónica de G. Castiglione}

Aun después de lo expuesto, hay que insistir en que es posible que no tengamos un argumento contundente para demostrar que el conjunto de fenómenos que definen lo que llamamos mundialización se dieran en la obra de Castiglione. Sin embargo, en la intuición y la transformación intelectual del jesuita se generó la concepción de algunas manifestaciones artísticas posteriores; por ejemplo, hizo posible en Europa la expansión del concepto de monumentalidad de los grandes palacios asociados a elementos y materiales chinos, sin considerar que su

the European Section in the Yuanming Yuan)", The Yuanmingyuan Journal, vol. 3, 1984, pp. 21-24.

${ }^{22}$ Liuli es un término arquitectónico que se refiere a los azulejos de los palacios chinos. Véanse ibid., p. 21, y Wong, A Paradise Lost, op. cit., p. 59.

${ }^{23}$ El lago Taihu es uno de los mayores de agua dulce de China. Véanse Jin Yufeng, "Yuanming Yuan Lou Pingxi", op. cit., p. 21, y Wong, A Paradise Lost, op. cit., p. 59.

${ }^{24}$ Fragmento de la carta de V. Hugo al Capitán Butler. Victor Hugo, Oeuvres, Acts et paroles pendant l'exil, Manuscript, París, Biblioteca Nacional de Francia, Departamento de Manuscritos, NAF 243778, 1875. 
obra constituiría la posibilidad de una tendencia globalizadora. Nuestro interés está centrado en ayudar a comprender que la obra de Castiglione sigue siendo actual y podemos relacionarla con las conocidas chinoiseries de la Europa de los siglos XVIII y XIx. Esto es evidente, sobre todo, en un amplio espectro de monumentales obras arquitectónicas y actuaciones en jardines de tipo chinesco. (Otros investigadores utilizan los términos euroiseries y occidenteries ${ }^{25}$ para mostrar la participación de Europa en el desarrollo cultural y artístico de China a partir del siglo XVIII.) En China aparecieron las primeras influencias occidentales, o europeneeries, ${ }^{26}$ en las fuentes diseñadas por el jesuita M. Benoit para los palacios de estilo europeo. Una de ellas, quizá la más grandiosa, perteneciente al Palacio del Mar en Calma (Haiyantang), se comportaba como un original reloj de agua formado con las doce cabezas de animales del horóscopo chino. Estas representaciones sirvieron de modelo al artista contemporáneo Ai WeiWei para una exposición, en Nueva York, en 2011. Una de sus obras, conocida como Las doce cabezas del zodiaco, está inspirada en las cabezas originales de la fuente del palacio de estilo europeo Haiyantang.

Estos modelos innovadores ${ }^{27}$ adquirieron un auge extraordinario durante la pujante situación económica del reinado de Qianlong, y el gran avance tecnológico desarrollado por los arquitectos misioneros jesuitas. $\mathrm{Chen}^{28}$ asegura que derivan del propio concepto chino de monumentalidad, íntimamente relacionado con el de grandiosidad en edificios construidos en la China antigua. ${ }^{29}$ Parece que el conjunto de recintos de estilo europeo, fruto de la personalidad de G. Castiglione, tuvo una fuerte influencia en las chinoiseries que florecieron en Europa:

${ }^{25}$ Véase Kristina Kleutghen, “Chinese Occidenterie. The Diversity of 'Western' Objects in Eighteenth-Century China”, Eighteenth-Century Studies, vol. 47, núm. 2, invierno de 2014, pp. 117-135.

${ }^{26}$ George N. Kates, The Years that were Fat. Peking, 1933-1940, Nueva York, Harper \& Brothers Publishers, 1952, p. 198.

${ }^{27}$ Kristina Kleutghen, "Staging Europe, Theatricality and Painting at the Chinese Imperial Court”, Eighteenth Century Studies, vol. 42, 2013, pp. 81-102.

${ }^{28}$ Chen Fang, Study of Theatrical Hall in Beijing during Qianlong Period, Beijing, Wenhua yishu chubanshe, 2001, p. 65.

${ }^{29}$ Wang Guixiang, "Summarized Discussion on Development of Ancient Chinese Wooden Architecture”, Journal of Traditional Architecture and Gardens, núm. 2, 1985, pp. 3-15. 
FigURA 7. Ilantai (1749-1789), grabado $7(90 \times 50 \mathrm{~cm})$, "Yangque Long dongmian", Pictures of the European Palaces and Waterworks, 1781-1787, Bibliothèque Nationale de France, París

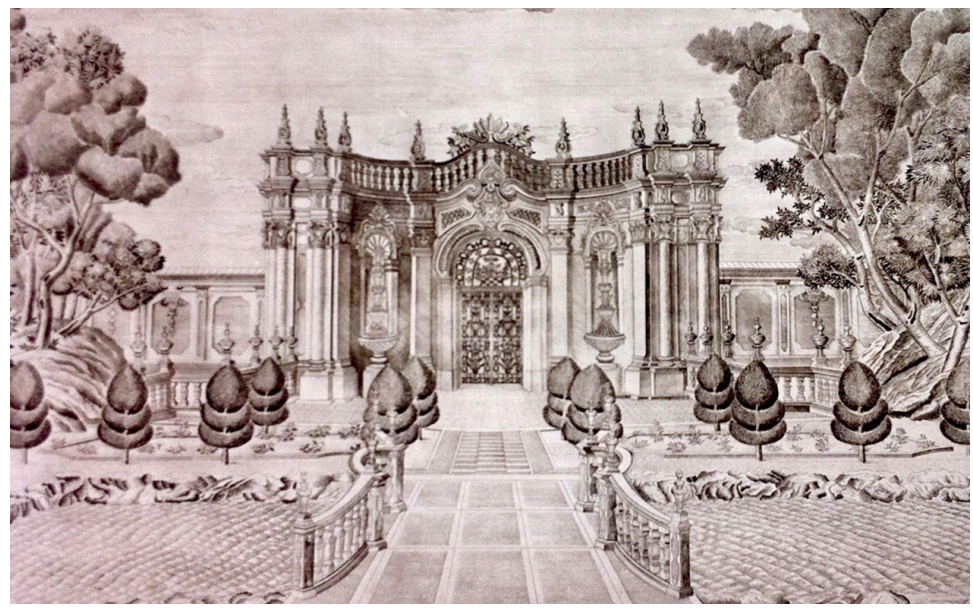

La Pagode de Chanteloup (1778); el Kiosque de Chantilly (1784); el Trianón de Versalles; el Pavillon Chinois de Drottningholm, en Suecia; la Sala de Porcelana del Palacio Real de Aranjuez (1763), en España; sin olvidar la Galería China del Royal Pavilion de Brighton (1819), en Gran Bretaña, con evidentes muestras de influencias chinas, entre otros. También el Palacio de Dolmabahçe, en Turquía, construido entre 1842 y 1853 , cuya decoración oriental y europea contiene elementos estilísticos muy diversos y una acusada influencia conceptual de los palacios de estilo europeo, como puede verse en las figuras 7 y 8. Sus complicados techos, adornados con exuberantes dorados, y sus paredes, decoradas con pinturas en perspectiva, crean la ilusión de una falsa continuidad arquitectónica. Desafortunadamente, algunos de estos edificios han desaparecido, y es prácticamente imposible determinar la parte que corresponde a cada civilización en la hibridación de formas y motivos occidentales y orientales. 
Figura 8. Palacio de Dolmabahçe, Estambul, Turquía, 1842-1853

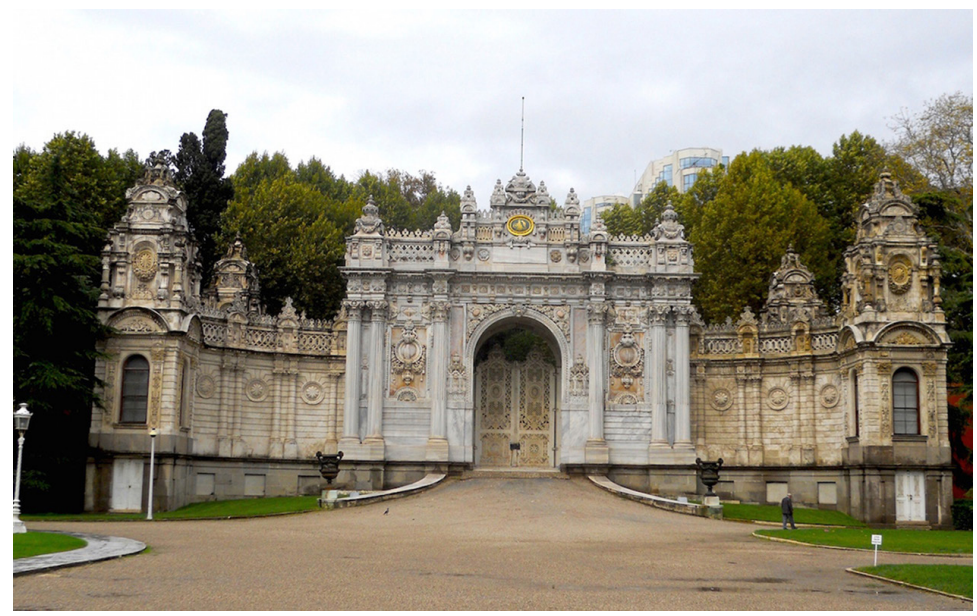

Fuente: dominio público.

Estas influencias orientales llegarían al estilo conocido como jardin inglés del siglo XvIII, en el que William Kent (16851748 ) intentó recrear ambientes nostálgicos e idílicos, entre 1730 y 1740. Influencias que no sólo implicaban elementos escogidos de la naturaleza, sino también arquitectura, escultura, alusiones a la pintura, la poesía y la mitología. Estos aspectos ornamentales del jardín kentiano no estaban limitados al mundo clásico, sino que estuvieron abiertos a elementos no europeos $\mathrm{y}$, por lo tanto, a la monumentalidad artística del siglo XVIII en los territorios asiáticos. Un ejemplo lo constituye la obra del arquitecto William Chambers (1723-1796), llegado en 1749 a la Escuela de Artes de París, en el momento en el que se publican las Lettres édifiantes et curieuses, en una de las cuales el misionero jesuita J. Denis Attiret describió los jardines del emperador de China en Beijing. ${ }^{30} \mathrm{El}$ interés despertado en el joven

${ }^{30}$ Jean Denis Attiret (frère Attiret), "Les Jardins Chinois”, en Lettres édifiants et curieuses de Chine par les missionnaires jésuites 1702-1776, París, Garnier-Flammarion, 1979, pp. 411-429. 
arquitecto lo llevó a publicar, en 1757, Designs of Chinese Buildings, Furniture, Dresses, Machines and Utensils, ${ }^{31}$ con gran éxito en Inglaterra y Francia, y en su Dissertation on Oriental Gardening, 32 de 1772, aumenta y amplía sus opiniones sobre el jardín. Así se expresa Chambers en el último capítulo de la primera de estas obras: "Los jardineros chinos, al igual que los pintores europeos, toman los objetos más placenteros de la naturaleza y procuran combinarlos de tal modo que no sólo se presenten de la manera más ventajosa por separado, sino que asimismo formen un conjunto elegante y sorprendente". Las chinoiseries eran tan especialmente atractivas, que en el jardín inglés generarían un ambiente oriental y exótico a la vez. Una de sus obras, Plans, Elevations, Sections and Perspective Views of the Gardens and Buildings at Kew in Surrey, ${ }^{33}$ muestra los jardines creados por Chambers en la década de 1760, en los que se incluyeron recintos arquitectónicos muy distintos; destacan una pagoda china y la Casa de Confucio (figura 9).

La pagoda no fue una copia de las que Chambers conoció en China, ya que le imprimió cierta personalidad en el contexto del jardín. ${ }^{34}$

Consecuentemente, G. Castiglione no representó la ruptura con la tradición, pero reivindicó la modernidad. Su modelo de hibridación tipológica, entre fantasía y realidad, China y Europa, fue una vez más un claro exponente de la fusión de las dos culturas y tradiciones en el contexto de una primera tendencia globalizadora. Es de admirar cómo Giuseppe Castiglione unió la acción misional, magistralmente desarrollada en la corte de Qianlong, con la vocación más trascendental de las ciencias y las artes: el sentido de servicio.

${ }^{31}$ William Chambers, Designs of Chinese Buildings, Furniture, Dresses, Machines and Utensils, Nueva York, Arno Press, 1980.

${ }^{32}$ William Chambers, Dissertation on Oriental Gardening, Londres, W. Griffin, 1772.

${ }^{33}$ William Chambers, Plans, Elevations, Sections and Perspective Vieres of the Gardens and Buildings at Kew in Surrey, Londres, Gregg Press, 1966.

${ }^{34}$ Manuel V. Castilla, "Vistas a un mar distante (Yuanying Guan): composición de la arquitectura moderna en los trópicos", tesis de doctorado de historia y composición arquitectónica, Universidad de Sevilla, Sevilla, 2013, pp. 224-228. 
Figura 9. W. Chambers, La Casa de Confucio, Plans, Elevations, Sections and Perspective Views of the Gardens and Buildings at Kew in Surrey, 1763

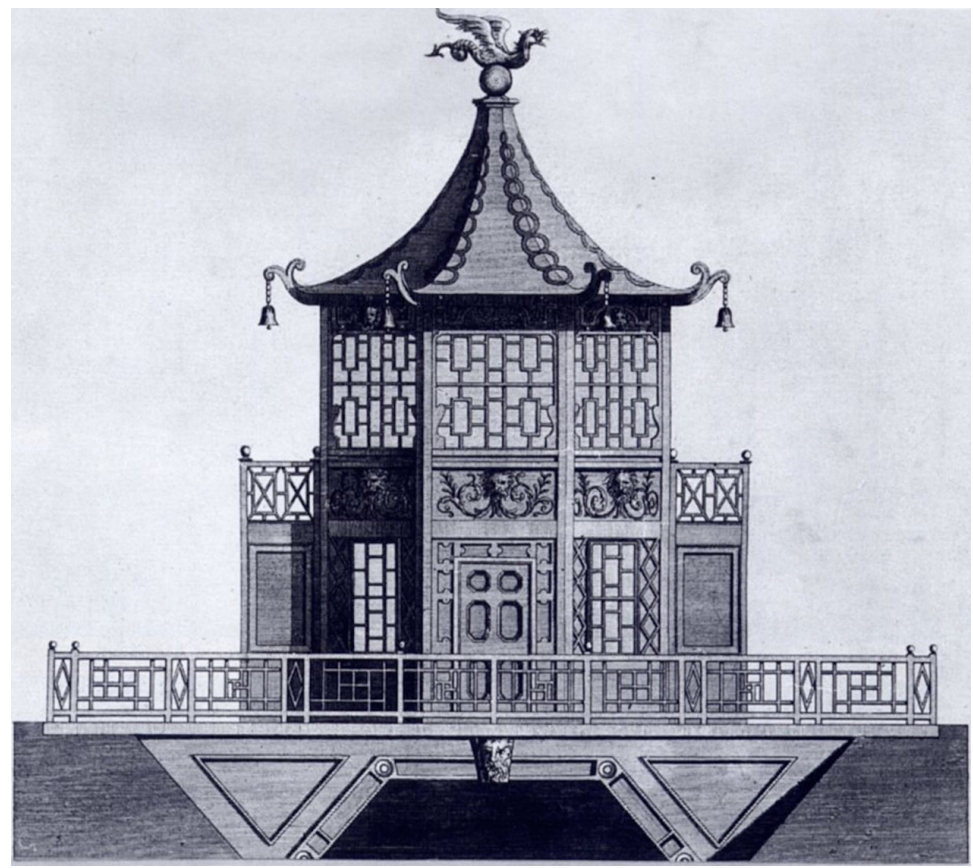

\section{Conclusión}

En este artículo se pone de manifiesto que el desarrollo epistemológico y práctico de la perspectiva matemática lineal realizado por el jesuita Giuseppe Castiglione, desde su privilegiada posición en la corte imperial china, jugó un papel fundamental en la hibridación cultural y artística en la China del siglo XVIII. La maestría del jesuita en aquella disciplina logró hacer convivir armoniosamente conceptos, temas, estilos y materiales de la pintura y la arquitectura china, con las estrategias y las técnicas del arte occidental. Al seguir las enseñanzas de su maestro, Andrea Pozzo, atribuyó a la perspectiva su estatus de ciencia 
matemática, y labró un espacio a través del cual organizó y transmitió el nuevo conocimiento científico-artístico, que él mismo creó, con una clara intencionalidad apostólica. Esta hibridación constituyó lo que podríamos entender como una primera mundialización cultural y artística, que con una intensidad inusitada es analizada en la actualidad por científicos e historiadores.

La presencia del monumentalismo simbólico del Yuanming Yuan en la arquitectura occidental se debe, en gran parte, a la contribución de G. Castiglione a la pintura y la arquitectura asiáticas gracias a su formación matemática, con el proyecto y la construcción de los palacios de estilo europeo. Su modelo de combinación tipológica China-Europa fue, una vez más, un claro exponente de la fusión de las dos culturas y tradiciones. Toda la corte del emperador admiró los efectos teatrales de la perspectiva en sus proyectos y sus pinturas, y es el primer jesuita que articuló la idea del diseño y la geometría proyectiva en sus obras.

Castiglione fue un extraordinario artista y atento observador, además de humilde misionero jesuita. Su capacidad de comprensión y adaptación a la cultura y las artes chinas provocó el entendimiento y la fascinación de Europa por ellas; sin embargo, a pesar de su extraordinaria aportación cultural, artística y arquitectónica en Asia Oriental, en Occidente sigue siendo un gran desconocido. Hasta el siglo XX no ha sido conocida ni reconocida su obra fuera de China, ni como arquitecto ni como pintor de interiores en los palacios de estilo europeo. Consideramos que las razones para este desconocimiento han sido varias, pero la más simple, la que algunos llaman razón occidental, consiste en que los occidentales no han reconocido ni sus técnicas ni los proyectos en los que participó. En su tiempo, el jesuita italiano, visto desde Occidente, era totalmente chino, y en cierto modo lo era, ya que desde que Castiglione se instaló en Beijing, su arte y su imaginación estuvieron fuertemente influidos por el país asiático. Paralelamente, sus magistrales diseños, con gran profusión de ornamentos barrocos, fueron muy representativos de la estética chino-europea. Los palacios de estilo europeo del Yuanming Yuan no sólo fueron una herramienta misionera, utilizada en la estrategia de acomodo introducida por el padre Ricci, sino también un símbolo del exotismo 
europeo triunfante en la corte china del siglo XVIII, exportado después a la arquitectura europea.

Dirección institucional del autor:

Universidad de Sevilla

CL. Virgen de África 7

41011, Sevilla, España

\mviggo@us.es

\section{Bibliografía}

Attiret, J. Denis (frère Attiret), "Les Jardins Chinois", en Lettres édifiants et curieuses de Chine par les missionnaires jésuites 17021776, París, Garnier-Flammarion, 1979.

Beurdeley, Cécile y Michel Beurdeley, Giuseppe Castiglione. A Jesuit Painter at the Court of the Chinese Emperors, trad. Michael Bullock, Rutland-Tokio, Tuttle y Co., 1971.

Beurdeley, Michel y Marco Musillo, Peintres Jésuites en China au XVIII siècle, París, Anthèse, 1997.

BotTon Beja, Flora, "Reseña del libro de Elisabetta Corsi, La fábrica de las ilusiones. Los jesuitas y la difusión de la perspectiva lineal en China (1698-1766)", Estudios de Asia y África, vol. XL (2), núm. 127, 2005, pp. 465-469.

Castilla, Manuel V., "Vistas a un mar distante (Yuanying Guan): composición de la arquitectura moderna en los trópicos", tesis de doctorado de historia y composición arquitectónica, Universidad de Sevilla, Sevilla, 2013.

Corsi, Elisabetta, "Perspectiva iluminadora e iluminación de la perspectiva. La versión del arte occidental de la perspectiva de Nian Xiyao (1671-1738) en los prólogos a la 'Ciencia de la visión'”, Estudios de Asia y África, vol. xxxvi (3), núm. 116, 2001, pp. 375-418.

CoRsI, Elisabetta, "Términos chinos utilizados como sinónimos de 'perspectiva' y ecos del naturalismo en algunos textos sobre pintura del siglo XVIII", Estudios de Asia y Africa, vol. XXXII (3), núm. 104, 1997, pp. 433-460.

Chambers, William, Designs of Chinese Buildings, Furniture, Dresses, Machines and Utensils, Nueva York, Arno Press, 1980.

Chambers, William, Dissertation on Oriental Gardening, Londres, W. Griffin, 1772. 
Chambers, William, Plans, Elevations, Sections and Perspective Vieros of the Gardens and Buildings at Kew in Surrey, Londres, Gregg Press, 1966.

CHEN, Fang, Study of Theatrical Hall in Beijing during Qianlong Period, Beijing, Wenhua yishu chubanshe, 2001.

De Feo, Vittorio y Martinelli Vittorio, Andrea Pozzo, Milán, Electa, 1996.

FERRER, José Luis, La perspectiva en las artes y en la técnica, Valencia, Universidad Politécnica de Valencia, 1981.

Guixiang, Wang, "Summarized Discussion on Development of Ancient Chinese Wooden Architecture", Journal of Traditional Architecture and Gardens, núm. 2, 1985, pp. 3-15.

Hugo, Victor, Oeuvres, Acts et paroles pendant l'exil, Manuscript, París, Biblioteca Nacional de Francia, Departamento de Manuscritos, NAF 243778, 1875.

Kates, George N., The Years that were Fat. Peking, 1933-1940, Nueva York, Harper \& Brothers Publishers, 1952.

Kemp, Martin, La scienza dell'arte. Prospettiva e perzecezione visiva da Brunelleschi a Seurat, trad. F. Camerota, Florencia, Giunti, 1994.

Kleutghen, Kristina, "Chinese Occidenterie. The Diversity of 'Western' Objects in Eighteenth-Century China”, EighteenthCentury Studies, vol. 47, núm. 2, invierno de 2014, pp. 117-135.

Kleutghen, Kristina, "Staging Europe, Theatricality and Painting at the Chinese Imperial Court", Eighteenth Century Studies, vol. 42, 2013, pp. 81-102.

Marinescu, Joselyn M. N., "Defending Christianity in China: The Jesuit Defense of Christianity in the Lettres édifiantes et curieuses \& Ruijianlu in relation to the Yongzheng Proscription of 1724", tesis de doctorado, Kansas State University, Manhattan, 2008.

Meer Harold, Alan, Guarino Guarini and his Architecture, New Haven-Londres, Yale University, 1989.

Musillo, Marco, Bridging: Europe and China: The Professional Life of Giuseppe Castiglione (1688-1766), tesis de doctorado, University of East Anglia, Norwich, 2006.

Musillo, Marco, "Reconciling Two Careers: The Jesuit Memoir of Giuseppe Castiglione Lay Brother and Qing Imperial Painter”, Eighteenth-Century Studies, vol. 42, núm. 1, 2008, pp. 45-59.

Pelliot, Paul, Les Influences Européennes Sur L'art Chinois, París, Imprimerie Nationale, 1928.

Pirazzoli-t'Serstevens, Michèle, Giuseppe Castiglione 1688-1766: Peintre et architecte à la cour de Chine, París, Thalia, 2007. 
Pozzo, Andrea, Prospettiva de'pittori e architetti, Roma, Italo Svevo, 2003.

Sierra de la Calle, Blas, Catálogo Colección S. C. Cheng de Arte chino, Valladolid, Museo Oriental-Caja España, 1999.

Sjönström, Ingrid, Quadratura: Studies in Italian Ceiling Painting, Estocolmo, Universidad de Estocolmo, 1978.

Wong, Young-Tsu, A Paradise Lost. The Imperial Garden Yuanming Yuan, Honolulú, Universidad de Hawái, 2001.

Yufeng, Jin, "Yuanming Yuan Xiyang Lou Pingxi (A Critical Analysis of the European Section in the Yuanming Yuan)", The Yuanmingyuan Journal, vol. 3, 1984, pp. 21-24. 